\title{
Manufacturing of Silica Glass from Silica Power Consolidated with Tetramethoxysilane Oligomer-derived Binder
}

\author{
Osamu YAGI \\ Tama chemicals Co., LTD. (3-22-9 Shiohama, Kawasaki-ku, Kawasaki, Kanagawa 210-0826, Japan) \\ Present Address: Chouetsushi Laboratory, Kazari-ichi Co., Ltd. (Room no. F, Sangaku Kyodo Kenkyu Center, 1-1-2 \\ Suehiro-Cho, Tsurumi-ku, Yokohama, Kanagawa 230-0045, Japan) \\ Received September 3, 2001 ; Accepted January 10, 2002

\begin{abstract}
A sol-gel method was used for manufacturing silica glass. A new method involved making a bulk by hardening a slurry of silica powder with a methoxysilane-derived binder. The bulk manufactured by this method was successfully dried and sintered to produce the silica glass. The significant results of this method are as follows: (1) the amount of reaction water in synthesizing the binder was less than the amount with which the methoxy group in the methoxysilane was completely hydrolyzed and poly-condensed, (2) the methoxy group partially survived in the binder and (3) this survival caused the binder to be flexible. When an oligomer of tetramethoxysilane was used as a starting material, the optimum amount of water for the bulk manufacturing is 2.3 equivalents. The bulk manufactured by this method was dried and sintered to form the purified silica glass without the generation of cracks.
\end{abstract}

Key Words : Sol-gel Method, Silica Crlass, Tetramethylammonium Hydroxide, Tetramethoxysilane

\section{Introduction}

Silica glass is one of the most important materials for semiconductor manufacturing, because it has a high melting point and a small amount of metallic impurities. In general, the silica glass used for manufacturing a large size wafer is synthesized by melting a natural one which has a large amount of metallic impurities such as $\mathrm{Al}$ and Ti. The sol-gel method ${ }^{1,2)}$ has been widely applied to the manufacturing of silica glass but has been mostly unsuccessful, because the green compact must shrink and therefore produce many cracks during the drying process. ${ }^{3)}$ Many methods were tried to reduce the shrinkage, in which colloidal silica was mixed in the sol solution as an anti-shrinkage agent." This method was much useful to reduce the shrinkage, however, a precipitation of colloidal silica took place when the sol solution was stood for a long time, and this made the gel generate many cracks during the drying process.

In this paper, a new method for the effective and inexpensive manufacturing of purified silica glass is described. The sol-gel method of hardening a slurry of a purified synthetic silica powder with a binder has been applied to prevent cracking of the silica bulk during the drying process and then the bulk was successfully sintered to produce the silica glass. The synthetic silica powder is purer than a natural one. To prevent cracking during the drying process, the binder must be flexible. In this paper, a synthetic method for the binder to be flexible enough and a hardening method for the slurry of a silica powder with the binder without the generation of cracks are described.

\section{1 Materials}

\section{Experimental}

Methanol and ethanol were both distilled from the extra pure grade reagents (Kanto Chemical Co., Inc.) and stored in a polyethylene bottle. Ethylene glycol and ammonia water (the special grade reagents both from Kanto Chemical Co., Inc.) were used without further purification. The oligomer of tetramethoxysilane (MS-51: trimer as the mean polymerization degree) and tetramethylammonium hydroxide (TMAH) solution were both made by Tama Chemicals Co., Ltd. The silica powder $(20 \mu \mathrm{m}$ mean diameter) was made by Mitsubishi Rayon Co., Ltd. 2. 2 Decomposition temperature of methoxy group measured by the differential thermal analysis method

The sample was prepared as follows. In a $500 \mathrm{ml}$ three-necked glass vessel equipped with a condenser and a thermometer, $218.4 \mathrm{~g}$ of MS-51 and $80 \mathrm{~g}$ of methanol were added and stirred, and then $16.2 \mathrm{~g}$ of a $0.05 \%$ TMAH aqueous solution was added as the reaction water and catalyst. The reaction mixture was heated in an oil bath at $72^{\circ} \mathrm{C}$ for $2 \mathrm{~h}$. After cooling, $64.5 \mathrm{~g}$ of the reaction mixture was mixed with $22.0 \mathrm{~g}$ of ethanol and $20.0 \mathrm{~g}$ of ethylene glycol in a polyethylene vessel, and then stirred with $1.7 \mathrm{~g}$ of a $2 \%-\mathrm{NH}_{4} \mathrm{OH}$ solution for $4 \mathrm{~min}$, forming a gel without stirring after $10 \mathrm{~min}$ at room temperature. The decomposition temperature of the gel was measured by the differential thermal analysis method.

\section{3 Synthesis of the bulk}

In a $500 \mathrm{ml}$ three-necked quart $z$ vessel equipped with a condenser and a thermometer, $218.4 \mathrm{~g}$ of MS-51 and 80 $\mathrm{g}$ of methanol were added and stirred, and then a $0.05 \%$ TMAH aqueous solution was added as the reaction water and catalyst. The reaction mixture was heated in an oil 
bath to make the binder. After cooling, $64.5 \mathrm{~g}$ of the binder was mixed with ethanol and ethylene glycol in a polyethylene vessel, and then stirred with $250 \mathrm{~g}$ of the silica powder to make a slurry. The slurry was mixed with $1.7 \mathrm{~g}$ of a $2 \%-\mathrm{NH}_{4} \mathrm{OH}$ solution and stirred for $4 \mathrm{~min}$. It was then poured into the cylindrical mold with a 105 $\mathrm{mm}$ diameter which was covered with a polyethylene sheet. After $3 \mathrm{~h}$, the hardened wet bulk was removed from the mold and put into a polyvinyl chloride bag. Five small holes of about $5 \mathrm{~mm}$ diameter each were made in it every $24 \mathrm{~h}$. The bulk which was dried in this way for 1 week was then allowed to stand in open air.

\section{Results and Discussion}

The most important problem when using the sol-gel method for synthesizing the binder for hardening the powder is to prevent cracking during the drying process. Crack formation in the gel is mainly caused by the increased of the capillary force, ${ }^{1 /} \Delta \mathrm{P}$ (Eq. (1)), as a result of a vaporation of the solvents contained in the gel.

$$
\Delta \mathrm{P}=2 \gamma \cos \Theta / \mathrm{r}
$$

where $r$ is the capillary diameter, $\gamma$ is the surface tension, and $\Theta$ is the contact angle between the capillary walls and the solvent.

When a thin film is prepared by hydrolysis and polycondensation of tetramethoxysilane $\left(\mathrm{Si}\left(\mathrm{OCH}_{3}\right)_{4}\right.$ (1)), it will be hard because it only consists of the siloxane bond $(\equiv \mathrm{Si}-\mathrm{O}-\mathrm{Si} \equiv)(\mathrm{Eq} .(2))$. This bond is too rigid for the film to prevent the generation of cracks. This is the reason why the thick film cannot be easily obtained when tetramethoxysilane is used as the starting material. On the other hand, the film prepared with alkyltrimethoxysilane $\left(\mathrm{RSi}\left(\mathrm{OCH}_{3}\right)_{3}\right.$ (4)) contains an unhydrolyzed alkyl group $(\equiv \mathrm{Si}-\mathrm{R})$ even if the methoxy groups are completely hydrolyzed (Eq. (3)).

$\mathrm{Si}\left(\mathrm{OCH}_{3}\right)_{4}+2 \mathrm{H}_{2} \mathrm{O} \stackrel{\mathrm{H}^{+} \text {or } \mathrm{OH}^{-}}{\longrightarrow} \mathrm{SiO}_{2}+4 \mathrm{CH}_{3} \mathrm{OH}$

(1)

(2) (3)

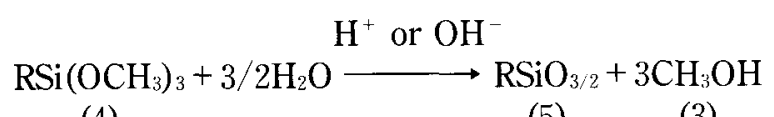

(4)

(5)

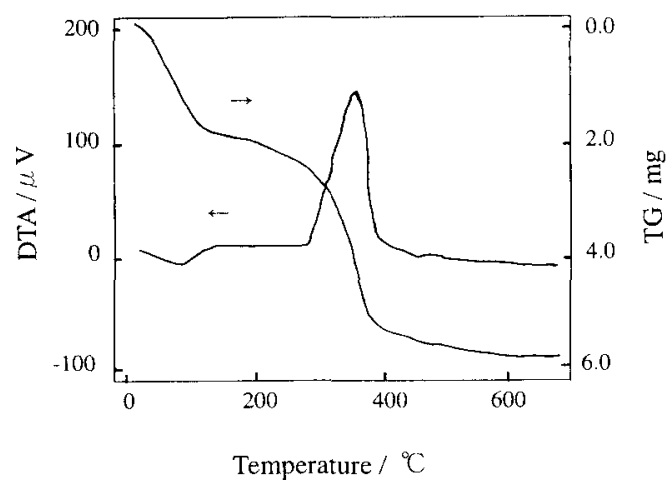

This film is flexible enough to absorb the capillary force, $\Delta \mathrm{P}$. This is the reason why the thick film can be easily obtained when they are used as a starting material. ${ }^{4}$ This indicates that no crack should be generated in the binder if the unhydrolyzed group remains after the hydrolysis and poly-condensation of methoxysilane.

Alkyltrimethoxysilane is used as the starting material, however, the survival of the alkyl group must occur in the film, and which is carbonized at about $600^{\circ} \mathrm{C}^{5}$ ) when the film is sintered. The most favorable binder is one that behaves in the hydrolysis process as an alkyltrimethoxysilane which is very flexible to absorb the shrinkage during the drying process, on the other hand, it behaves in the sintering process as a tetramethoxysilane which only provides of the siloxane bond after sintering.

To synthesize this type of binder, the amount of reaction water must be less than the equivalent one in which the methoxy groups of methoxsilane are entirely hydrolyzed to siloxane bond ( $\equiv \mathrm{Si}-\mathrm{O}-\mathrm{Si} \equiv$ ). In this case, the survived methoxy groups cannot be entirely hydrolyzed, and this survival will provide flexibility to the binder like the alkyl group of the alkyltrimethoxysilane.

If the methoxy groups remaining in the gel decompose to generate siloxane bonds at a relatively low temperature (Eq. (4)), the structure after sintering will be similar to that produced by the complete hydrolysis of tetramethoxysilane.

$2 \equiv \mathrm{Si}-\mathrm{OCH}_{3} \stackrel{\text { heat }}{\longrightarrow}: \equiv \mathrm{Si}-\mathrm{O}-\mathrm{Si} \equiv+$ Decomposition products

The decomposition temperature of the methoxy group in the gel was examined using the differential thermal analysis method. The result is shown in Fig. 1, indicating the decomposition under $600^{\circ} \mathrm{C}$. The color of sample after the pyrolysis was white and no carbon was detected. This result suggests that the remaining methoxy group changed to siloxane bond after heating.

The binder was synthesized as follows (Eq. (5)).

$$
\underset{(9)}{\mathrm{MS} 51}+\mathrm{nH}_{2} \mathrm{O}+\underset{(10)}{\text { Catalyst }} \stackrel{\text { reflux } / \mathrm{CH}_{3} \mathrm{OH}}{\longrightarrow} \text { Binder }
$$

The starting material was the oligomer of tetramethoxysilane (9) (MS-51: trimer as the mean polymerization degree). A catalyst for synthesizing the binder was tetramethylammonium hydroxide (TMAH) ${ }^{6}{ }^{6}$ which is an organic base as strong as $\mathrm{NaOH}$ or $\mathrm{KOH}$ and decomposes at $135 \sim 140^{\circ} \mathrm{C},{ }^{7)}$ leaving no decomposition residue and inorganic ions. As MS-51 used here is a trimer as the mean polymerization degree, 4 equivalents of water is consumed for complete hydrolysis and polycondensation to generate siloxane bonds (Eq. (6)).

Fig. 1 DTA and TG analyses of the gel synthesized from tetramethoxysilane. 
Table 1 Influence of water content on binder and bulk syntheses.

\begin{tabular}{|c|c|c|c|c|c|c|c|c|}
\hline \multirow{2}{*}{ Run } & \multirow{2}{*}{$\begin{array}{c}\text { MS-51 } \\
\mathrm{g}\end{array}$} & \multicolumn{2}{|c|}{$\mathrm{H}_{2} \mathrm{O}^{\mathrm{a}}$} & \multirow{2}{*}{$\begin{array}{c}\text { Methanol } \\
g\end{array}$} & \multirow{2}{*}{$\underset{\mathrm{h}}{\operatorname{Time}^{c}}$} & \multirow{2}{*}{$\begin{array}{l}\text { Binder } \\
\text { catalyst }^{\text {t }}\end{array}$} & \multirow{2}{*}{$\begin{array}{c}\mathrm{H}_{2} \mathrm{O}^{\mathrm{e}} \\
\text { Equivalent }^{\mathrm{b}}\end{array}$} & \multirow{2}{*}{ Result $^{f}$} \\
\hline & & $g$ & Equivalent $^{\mathrm{b}}$ & & & & & \\
\hline 1 & 218.4 & 21.6 & 2.0 & 80 & 3 & TMAH & 2.8 & $\begin{array}{c}\text { Two short } \\
\text { cracks }\end{array}$ \\
\hline 2 & 218.4 & 16.2 & 1.5 & 80 & 7 & TMAH & 2.3 & No cracks \\
\hline 3 & 218.4 & 10.8 & 1.0 & 80 & 7 & TMAH & 1.8 & $\begin{array}{l}\text { One short } \\
\text { crack }\end{array}$ \\
\hline 4 & 218.4 & 8.6 & 0.8 & 80 & 14 & TMAH & 1.6 & $\begin{array}{l}\text { Many long } \\
\text { cracks }\end{array}$ \\
\hline
\end{tabular}

${ }^{a}$ Water content in the binder. ${ }^{b}$ Mole equivalent to the methoxy group in MS-51. "Refluxing time. ${ }^{\mathrm{d}} 0.05 \%$ solution. ${ }^{\mathrm{e}}$ Water content in the slurry after addition of a $2 \%-\mathrm{NH}_{4} \mathrm{OH}$ soln. $1.7 \mathrm{~g}$. Short crack: less than $10 \mathrm{~mm}$ depth, long crack: more than $10 \mathrm{~mm}$ depth.

Table 2 Composition of the silica slurry.

\begin{tabular}{lc}
\hline \multicolumn{1}{c}{ Component } & Weight $(\mathrm{g})$ \\
\hline Silica powder & 250 \\
Binder & 64.2 \\
Ethanol & 22.0 \\
Ethylene glycol & 20.0 \\
$2 \%-\mathrm{NH}_{4} \mathrm{OH}$ solution & 1.7 \\
\hline
\end{tabular}<smiles>COC(C)(O[GeH2]O[AsH2]O)[Si](C)(OC)OC</smiles>

(12)

The binders synthesized with less anount of water are shown in Table 1 . The slurry was mixed with the binder and silica powder as shown in Table 2 and Fig. 2. The binder synthesized with 1.5 equivalents of water exhibited better result than the others (see run 2 in Table 1), and the total amount of water in the slurry is 2.3 equivalents, which included the water of the hardening catalyst ( $2 \%-\mathrm{NH}_{4} \mathrm{OH}$ solution).

When the amount of water in the slurry is 1 equivalent, the generated siloxane polymer results in a linear type polymer, and 2 equivalent water results in the planar type polymer. More than 2 equivalent water generates three dimensional network of siloxane bonds. Little methoxy groups remain in the formed gel for the water above 3 equivalents and the synthesized binder reduced its flexibility. This indicates why 2.3 equivalent water amount is better than the others. The role of TMAH as a catalyst is under investigation. The bulk thus obtained by this method was dried at room temperature and then sintered to silica glass without cracks at about $1800^{\circ} \mathrm{C}$ (see Fig. 3). X-ray diffraction analysis indicated that the sintered silica glass contained no crystalline phase (see Fig. 4). The metallic impurities in the synthesized binder and the silica glass were shown in Table 3 , which indicates that the silica glass synthesized by this new method has a higher purify than a natural quartz.

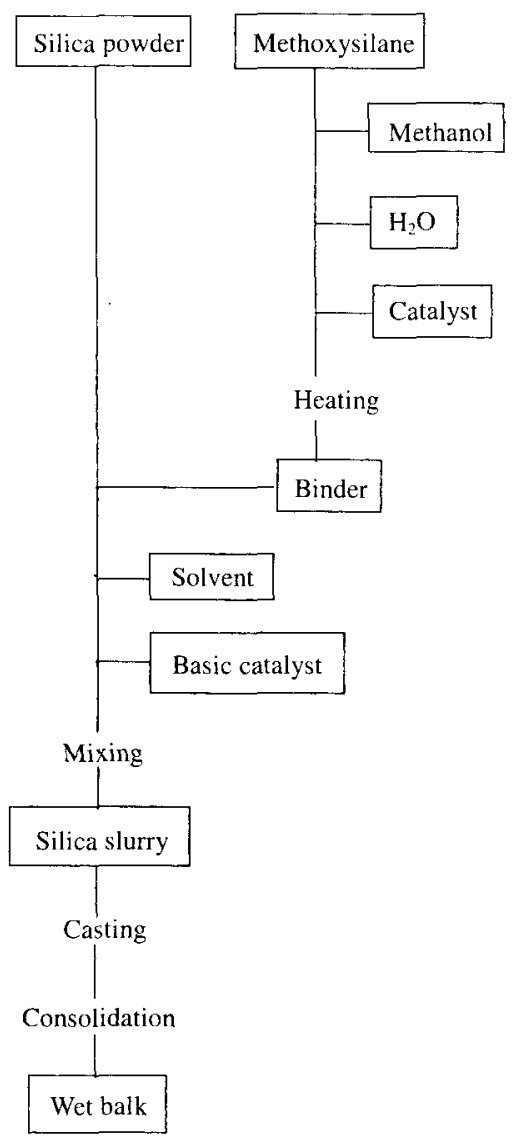

Fig. 2 Preparation procedure of wet bulk.

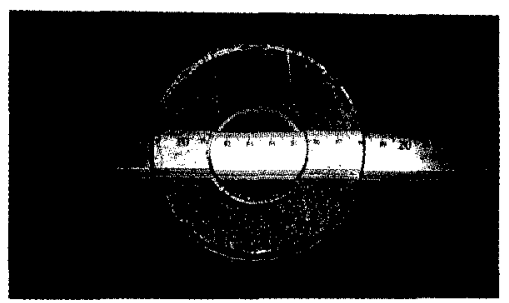

Fig. 3 Sintered bulk without generation of cracks.

\section{Conclusion}

A new method in which the silica powder slurry was hardened by the binder, was applied to manufacturing silica glass as follows: (1) the amount of reaction water 


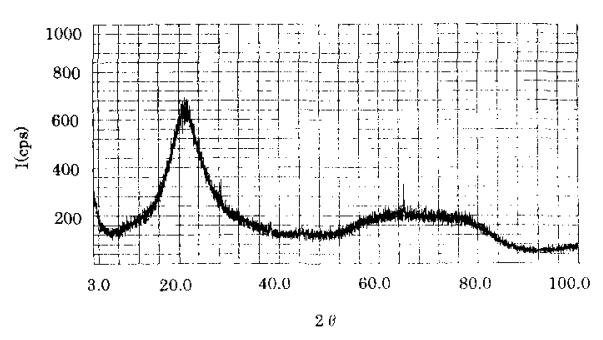

Fig. 4 X-ray diffraction of the sintered glass.

Table 3 Impurities of the binder, the sinterd glass and natural quartz.

\begin{tabular}{cccc}
\hline $\begin{array}{c}\text { Metal } \\
\text { ppm }\end{array}$ & Binder & Sinterd glass & Natural quartz \\
\hline $\mathrm{Na}$ & $<0.1$ & 0.2 & 1.0 \\
$\mathrm{~K}$ & $<0.1$ & 0.1 & 0.6 \\
$\mathrm{Fe}$ & $<0.1$ & 0.7 & 0.7 \\
$\mathrm{Al}$ & $<0.5$ & 0.3 & 17.7 \\
$\mathrm{Ti}$ & $<0.5$ & $<0.5$ & 1.1 \\
\hline
\end{tabular}

a) Natural quartz is produced by Unimin Corp. for synthesizing the binder was less than the amount for the complete hydrolysis and condensation of methoxy groups of methoxysilane, (2) the remaining methoxy groups gave the flexibility to the binder. The dried bulk was sintered to high purity silica glass without the formation of cracks.

\section{References}

1) C. J. Brinker and G. W. Scherer, Sol-Gel Science, Academic Press Inc. (1990).

2) L. L. Hench and J. K. West, Chem. Rev., 90, 33 (1990).

3) F. Kirkbir, H. Murata, D. Meyers, S. R. Chaudhuri, and A. Sarkar, J. Sol-Gel Sci. Tech., 6, 203 (1996).

4) M. Murakami, K. Izumi, T. Deguchi, and A. Morita, Nippon Seramikku Kyokai Gakujyutsu Ronbunshi, 97, 91 (1989).

5) K. Kamiya, T. Yoko, T. Sano, and K. Tanaka, J. Non-Cryst. Solids, 119, 14 (1990).

6) O. Yagi and S. Shimizu, Denki Kagaku (presently Electrochemistry), 64, 225 (1996).

7) W. K. Musker, J. Am. Chem. Soc., 86, 960 (1964). 